EPJ Web of Conferences 59, 18003 (2013)

DOI: $10.1051 /$ epjconf/20135918003

(C) Owned by the authors, published by EDP Sciences, 2013

\title{
Efficient multi-keV X-ray generation from high-contrast laser plasma interaction
}

Z. Zhang ${ }^{1, a}$, H. Nishimura ${ }^{1}$, M. Nishikino ${ }^{2}$, A. Sunahara ${ }^{3}$, T. Johzaki ${ }^{1}$, H.B. Cai ${ }^{4}$, T. Kawachi' ${ }^{2}$ A.S. Pirozhkov ${ }^{2}$, A. Sagisaka ${ }^{2}$, S. Orimo ${ }^{2}$, K. Ogura ${ }^{2}$, A. Yogo ${ }^{2}$, Y. Okano ${ }^{5}$, S. Ohshima ${ }^{1}$, S. Fujioka ${ }^{1}$, H. Kiriyama ${ }^{2}$, K. Kondo², T. Shimomura ${ }^{2}$ and S. Kanazawa ${ }^{2}$

1 Institute of Laser Engineering, Osaka University, 2-6 Yamada-oka, Suita, Osaka 565-0871, Japan

2 Quantum Beam Science Directorate, Kansai Photon Science Institute, JAEA, Kyoto 619-0215, Japan

3 Institute for Laser Technology, 2-6 Yamada-oka, Suita, Osaka 565-0871, Japan

4 Institute of Applied Physics and Computational Mathematics, PO Box 8009, Beijing 100088, China

${ }^{5}$ Laser Research Center for Molecular Science, Institute for Molecular Science, National Institute of Natural Science, 38 Nishigo-Naka, Myodaiji, Okazaki 444-8585, Japan

\begin{abstract}
K} \alpha$ line emission from Mo was experimentally and theoretically studied using clean, ultrahighintensity femtosecond laser pulses. The absolute yields of $\mathrm{K} \alpha \mathrm{x}$-rays at $17 \mathrm{keV}$ from Mo were measured as a function of the laser pulse contrast ratio and irradiation intensity. Significantly enhanced K $\alpha$ yields were obtained by employing high contrast ratio at optimum irradiance. Conversion efficiencies of $4.28 \times 10^{-5} / \mathrm{sr}$, the highest values obtained to date, was demonstrated with contrast ratios in the range of $10^{-10}$ to $10^{-11}$.
\end{abstract}

\section{INTRODUCTION}

As a bright, narrow band, energetic x-ray source, laser driven $\mathrm{x}$-ray is adapted for various applications including probing of dense matters treated in the field of high energy density physics [1]. Moreover, laser driven x-ray sources have been widely used for inner shell photoionization [2], ultra-fast biomedical imaging, lattice dynamics probing, and basic research in radiation biology.

A compact laser-driven $\mathrm{x}$-ray source with a high conversion efficiency $\left(\eta_{K \alpha}\right)$ is essentially needed for these applications. There are two key factors for achieving efficient $K \alpha$ line generation as a result of collisional ionization of inner-shell via energetic electron penetration in a matter: the energy distribution of the electrons (usually characterized by the hot electron temperature $\left(T_{h}\right)$ ) and transfer efficiency $\left(\eta_{T E}\right)$ from laser energy to hot electrons. Numerous studies have investigated the physics of hot electron generation and laser absorption. Both factors are critically affected by the formation of pre-plasma generated by a leakage pre-pulse from the laser system $[3,4]$. Consequently, the $K \alpha$ yield is substantially affected by pre-plasma formation [5].

In the present study, hard $\mathrm{x}$-rays at $17 \mathrm{keV}$ were generated from Mo targets. The target was irradiated by an intense laser pulse with an extremely suppressed pre-pulse. A two-stage pre-plasma with very

\footnotetext{
${ }^{a}$ e-mail: zhang-z@ile.osaka-u.ac.jp
}

This is an Open Access article distributed under the terms of the Creative Commons Attribution License 2.0, which permits unrestricted use, distribution, and reproduction in any medium, provided the original work is properly cited. 


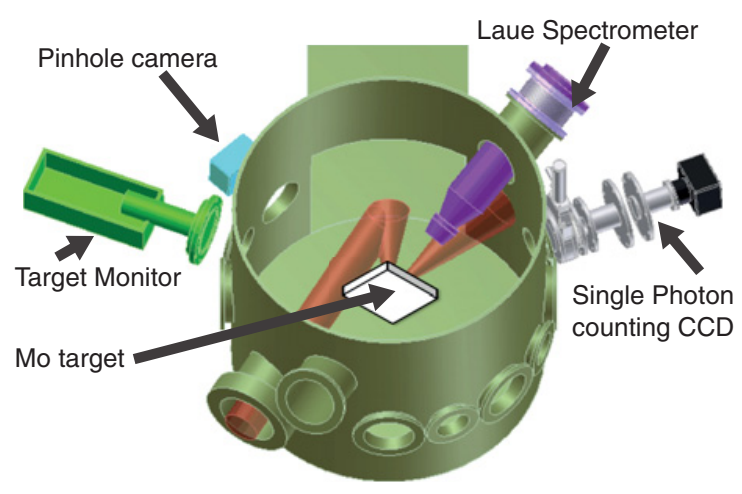

Figure 1. Experimental setup.

steep and moderate density scale lengths was formed and led to a combination of several absorption mechanisms. As a result, the highest $\eta_{K \alpha}$ of $4.28 \times 10^{-5} / \mathrm{sr}$ was achieved [6].

\section{EXPERIMENTAL SETUP}

Experiments were performed at J-KAREN laser facility at Japan Atomic Energy Agency, Kansai Photon Science Institute. This system delivers laser pulses at a wavelength of $800 \mathrm{~nm}$. The effective pulse duration is $58 \mathrm{fs}$, which provides the peak power of $30 \mathrm{TW}$ for a pulse energy of $1.8 \mathrm{~J}$. The laser pulse from the J-KAREN system contains two pre-pulse components: a leakage component from the preamplification stage, which has a flat profile and onsets $500 \mathrm{ps}$ before the main pulse, and a pedestal component caused by optical parametric chirped pulse amplifier (OPCPA) pump-induced noise and random spectral phase noise. This pedestal grows exponentially in time from the leakage component level to the foot of the main pulse. The component from $-500 \mathrm{ps}$ to $-100 \mathrm{ps}$ for $10^{-10}$ contrast is an order of magnitude greater than that for a contrast of $10^{-11}$. Refer to Refs. [7, 8] for further details for the pulse shape. To investigate the influence of the contrast ratio on the $\eta_{K \alpha}$, the contrast ratio of the leakage pulse was varied from $10^{-7}$ to $10^{-11}$ by controlling the operation conditions of the pulse cleaner and the optical shutters in the front-end stage of the system. An f/2.67 gold-coated off-axis parabolic mirror was used to focus a $p$-polarized laser beam at an incident angle of $22.5^{\circ}$ relative to the target normal, as shown in Fig. 1. 100- $\mu$ m-thick Mo planar targets were mounted on a motorized translation stage. The focal spot size was varied by translating the target along the laser beam, while the total energy at the target remained constant. In this way, the average laser intensity on the target was varied from $1 \times 10^{16}$ to $4 \times 10^{19} \mathrm{~W} / \mathrm{cm}^{2}$.

\section{EXPERIMENTAL RESULTS}

Figure 2 shows the $\eta_{K \alpha}$ per unit solid angle. In order to investigate the effect of contrast ratio, we first studied the $\mathrm{X}$-ray yield for a poor contrast ratio case $\left(10^{-7}\right.$; a typical contrast ratio in most chirped pulse amplifier (CPA) systems) as a reference. A strong dependence of $\mathrm{x}$-ray yield on the contrast ratio is observed: $\eta_{K \alpha}$ increases with improving contrast ratio. The asymmetry about the best focus is not fully understood yet, but a nonuniform intensity distribution on target may affect the overall $\mathrm{x}$-ray generation through the dependence of laser-plasma interaction and the hot electron velocity distribution on the local laser intensity. For both positive and negative offsets, the x-ray yield increases with increasing laser intensity. 

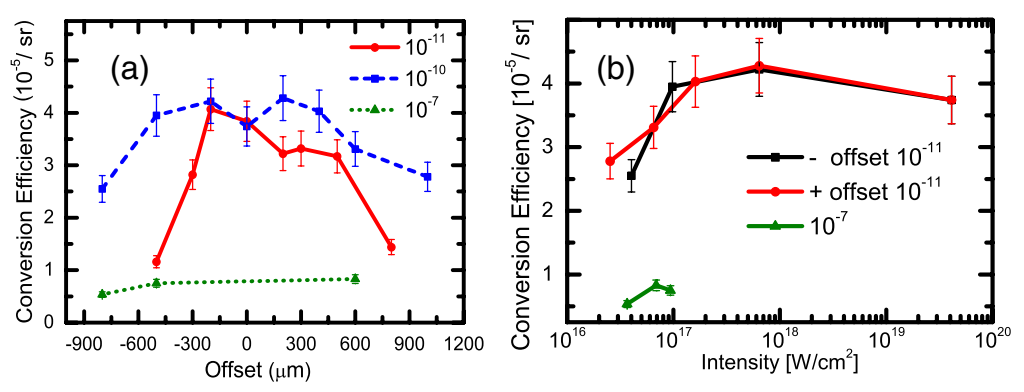

Figure 2. Mo K $\alpha$ yield: (a) as a function of offset, positive offset means the distance from the focusing mirror to the target is larger than the focal length; (b) as a function of intensity with contrast of $10^{-10}$.
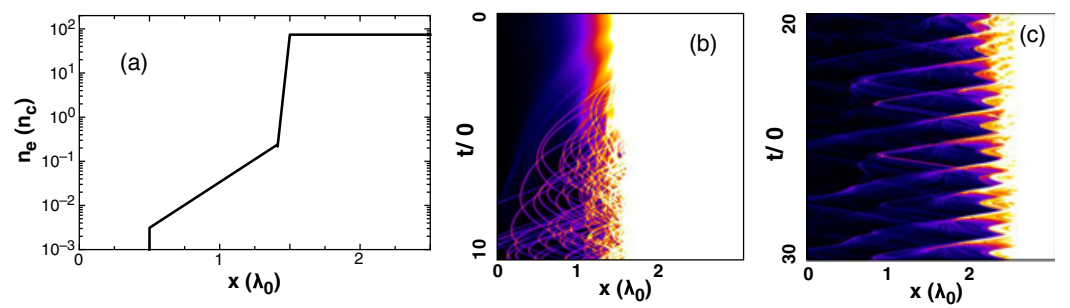

Figure 3. PIC simulation: (a) Initial density profile; (b) time evolution of the electron density for $\mathrm{t}=0-10 T_{0}$; (c) time evolution of the electron density for $\mathrm{t}=20 \mathrm{~T}_{0}-30 \mathrm{~T}_{0}$.

\section{SIMULATIONS}

The hot electron generation and laser absorption was theoretically studied. The preplasma with laser contrast of $10^{-10}$ and irradiation intensity of $4 \times 10^{19} \mathrm{~W} / \mathrm{cm}^{2}$ (best focus case) were simulated with a radiation hydrodynamic code [9]. A steep high density plasma with a long low density tail is formed, with density scale length $\mathrm{L}=0.03$ and $0.4 \lambda_{0}$ (where $\lambda_{0}$ denotes the laser wavelength), as shown in Fig. 3(a).

The laser plasma interaction simulation for the simulated preplasma was performed with a kinetic 1D particle-in-cell (PIC) code LPIC++ [10]. The oblique incident is treated using Bourdier's method [11]. The size of the simulation box is $200 \lambda_{0}$. The cell size is $\lambda_{0} / 100$ and totally 20000 cells are used. The time step used is $0.02 T_{0}$, where $T_{0}$ denotes the laser period and its value is $2.67 \mathrm{fs}$ for $800 \mathrm{~nm}$ laser wave. In order to set the preplasma and set enough number of particles per cell, a weighted particle method is used. In the first plasma ramp at the left side, we set 10 electrons and 10 ions per cell, in the second plasma ramp, we set 200 electrons and 200 ions per cell. For both the fields and particles, we use absorbing boundary condition. Based on the experimental condition, the simulation parameters were set with a laser incident angle of $22.5^{\circ}$ with $p$-polarization. The temporal shape of the laser pulse amplitude's envelope was characterized by sinusoidal edges with a rise time of $21.7 T_{0}$ and reached the peak intensity of $4 \times 10^{19} \mathrm{~W} / \mathrm{cm}^{2}$. Figure $3(\mathrm{~b})$ and (c) shows the temporal evolution of electron density for different time periods. We can see, at early stage, the front of laser pulse reaches the low density plasma and the electrons are pulled inside the high density region with ponderomotive force. Later, when the laser pulse propagates into the high density region, a part of the electrons are expelled into vacuum and gain energy in the laser field and the charge separation field. This is so called vacuum heating (VH) process and was first predicted by Brunel [12]. Meanwhile, the behavior of $\mathbf{J} \times \mathbf{B}$ heating can be seen at later stage, as shown in Fig. 3(c). From the simulation result, the laser transfer efficiency is $19.6 \%$. 


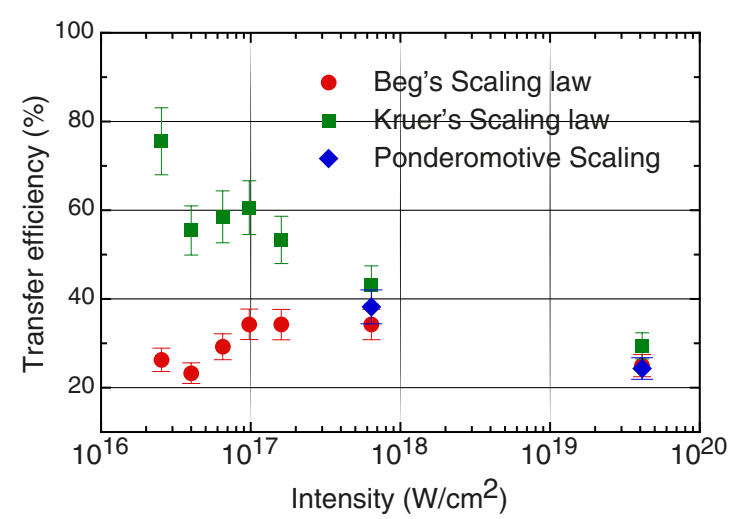

Figure 4. The $\eta_{T E}$ estimated from 3 scaling laws.

The simulation has also been made with a single slope preplasma case. For example for $\mathrm{L}=0.03 \lambda_{0}$, the transfer efficiency is about $9 \%$.

\section{TRANSFER EFFICIENCY}

According to D. Salzmann's model [13], the absolutely $\eta_{K \alpha}$ is given as a function of $\eta_{T E}$ and the hot electron temperature $T_{h}$ :

$$
\eta_{K \alpha}=\frac{\eta_{T E} n_{A} \omega_{K \alpha} E_{K \alpha}}{4 \pi T_{h}} \int_{0}^{\infty} d E \sigma_{K \alpha}(E) \int_{0}^{d} d x f_{h}\left(E_{0}, x\right) \exp \left(-\frac{x}{\lambda_{m f p} \cos (\theta)}\right)
$$

where $\sigma_{K \alpha}, \omega_{K \alpha}$ and $\mathrm{n}_{A}$ are, respectively, the cross section for $\mathrm{K}$-shell ionization, the $\mathrm{K} \alpha$ fluorescence yield, and the atomic number density. The term $\exp \left(-\frac{x}{\lambda_{m f p} \cos (\theta)}\right)$ describes the reabsorption of $\mathrm{K} \alpha$ photons during the propagation through the target material and $\theta$ refers to the angle from the spectrometer to target normal. By applying power scaling laws to estimate the $T_{h}$, the transfer efficiency $\eta_{T E}$ can be roughly derived from Eq. (1). For example, by applying the scaling law of Beg [14], Kruer [15] and also the ponderomotive law, the $\eta_{T E} \mathrm{~s}$ are estimated as shown in Fig. 4. According to Beg's law, the $\eta_{T E}$ in this experiment is about $30 \%$. In order to increase the accuracy of $\eta_{T E}$ estimation, a more precisely measurement of hot electron temperature is needed.

\section{CONCLUSION}

With quantitative $\mathrm{K} \alpha$ spectroscopy measurement, the behavior of laser plasma interaction with laser absorption and transfer have been experimentally and theoretically studied. The $\eta_{T E}$ of laser energy to hot electron is estimated to be $30 \%$. A two stage sharp preplasma is formed by the high contrast, high irradiance laser pulse. This two stage preplasma is possible to enhance the laser absorption and transfer efficiency [4], and results an enhancement of $\mathrm{K} \alpha \mathrm{X}$-ray yield.

The authors are indebted to the computer group at ILE and Cyber Media Center, Osaka University. This work was partly supported by the Common-Use Facility Program of JAEA. 


\section{References}

[1] R. Stephens, R. Snavely, Y. Aglitskiy, et al., Phys. Rev. E 69, 066414 (2004)

[2] S. Fujioka, H. Takabe, N. Yamamoto, et al., Nature Phys. 5, 821 (2009)

[3] M. Cerchez, R. Jung, J. Osterholz, et al., Phys. Rev. Lett. 100, 245001 (2008)

[4] H. B. Cai, K. Mima, A. Sunahara, et al., Phys. Plasmas 17, 023106 (2010)

[5] W. Lu, M. Nicoul, U. Shymanovich, A. Tarasevitch, et al., Phys. Rev. E 80, 026404 (2009)

[6] Z. Zhang, M. Nishikino, H. Nishimura, et al., Opt. Exp. 19, 4560 (2011)

[7] H. Kiriyama, M. Michiaki, Y. Nakai, et al., Appl. Opt. 49, 2105 (2010)

[8] H. Kiriyama, M. Mori, Y. Nakai, et al., Opt. Lett. 35, 1497 (2010)

[9] A. Sunahara, A Sasaki and K Nishihara, J. Phys.: Conference Series 112, 042048 (2008)

[10] R. Lichters, J. MeyerterVehn, and A. Pukhov, Phys. Plasmas 3, 3425 (1996)

[11] A. Bourdier, Phys. Fluids 26, 1804 (1983)

[12] F. Brunel, Phys. Rev. Lett. 59, 52 (1987)

[13] D. Salzmann, C. Reich, I. Uschmann, et al., Phys. Rev. E 65, 036402 (2002)

[14] F. Beg, A. Bell, A. Dangor, C. Danson, A. Fews, et al., Phys. Plasmas 4, 447 (1997)

[15] W. L. Kruer: The Physics of Laser Plasma Interactions (Addison-Wesley, 1988) 